\title{
In-Hospital Evolution of Non Operated Aneurysmal Subarachnoid Hemorrhage at the Neurology Unit of Befelatanana University Teaching Hospital, Antananarivo
}

\author{
Lemahafaka Jemissair Glorien, Neurologist \\ Rasaholiarison Nomena Finiavana, Neurology Resident \\ Rajaonarison Lala Andriamasinavalona, Neurologist \\ Razafimahefa Julien, Neurologist \\ Zodaly Noel, Neurologist
}

Tehindrazanarivelo Alain Djacoba, Professor of Neurology

Antananarivo Faculty of Medicine, University of Antananarivo, Madagascar

Doi: 10.19044/esj.2018.v14n24p148 URL:http://dx.doi.org/10.19044/esj.2018.v14n24p148

\begin{abstract}
Subarachnoid hemorrhage is defined as a sudden irruption of blood in subarachnoid spaces. This study aims to determine the profile and in-hospital evolution of patients with post aneurysmal subarachnoid hemorrhage, who did not have surgical treatment in the Neurology Unit of Befelatanana University Hospital, Antananarivo, Madagascar. This study was a descriptive retrospective study within a period of 4 years, from January 1st, 2014 to December 31th, 2017. The study was carried out in hospitalized patients for subarachnoid hemorrhage with aneurysmal origin. We included 52 patients, with an average age of 48 years and a female predominance. They are housewives in majority of cases. $52 \%$ of the cases live in urban areas while $77 \%$ of cases have high blood pressure. The main clinical manifestation was a sudden headache, and intracranial aneurysm was located at the anterior communicating artery in $52 \%$ of cases. All patients benefited from drug treatments in majority of cases. Due to the lack of financial means, $60 \%$ had neurological complications. However, in-hospital mortality rate was at $13 \%$. This study helps us to suggest to our patients medical treatment of their subarachnoid hemorrhage during hospitalization because of difficult access to surgery. It also aims to propose surgery outside the acute phase of the disease.
\end{abstract}

Keywords: Aneurysm, evolution, subarachnoid hemorrhage, surgery 


\section{Introduction}

Subarachnoid hemorrhage is defined as the presence of blood within the subarachnoid space. Its main aetiology is the rupture of intra-cranial vascular aneurysm in $85 \%$ of cases (Seiller et al., 2002; Swiss SOS study group, 2015).

It is a frequent pathology with an incidence of 7-10 cases out of 100,000 habitants per year in Europe. It constitutes a real neuro-vascular diagnosis and treatment emergency. The global prognosis of patients with post aneurysmal SAH stays unfavorable in spite of medicinal progress in these last decades. In addition, 1/3 of patients don't survive in the acute phase of the disease. Mortality rate stays high in hospital space and it reaches a mean of $50 \%$ of patients (Seiller et al., 2002; Swiss SOS study group, 2015).

The exclusion of the ruptured aneurysm should be in the first 72 hours by neuro-surgical way or endovascular way, thus allowing the prevention of precocious re-bleeding which is always bad (Fedoul et al., 2010). In Madagascar, surgical treatment for all patients is not always possible. Nowadays, knowledge about the initial gravity and the in hospital prognosis of non-operated aneurysmal SAH are limited. Thus, the interest and objective of our study is to determine the profile of patients who present aneurysmal $\mathrm{SAH}$, and to describe their evolution during hospitalization in the Neurology unit of Befelatanana University Hospital, Antananarivo.

\section{Materials and Method}

It is a retrospective study within a period of 4 years, from January 1 st 2014 to December 31th 2017, at the Neurology Unit of Befelatanana University Hospital, Antananarivo. Data were collected from hospitalized patients' records. This included all patients' records which show that diagnosis at discharge were post aneurysmal SAH without surgical or endovascular treatment, as well as clinical and radiological confirmation. All patients' records which were not completed or being out without medical advice were excluded.

Therefore, our study's variables were:

- Patients' demographic characteristics such as the age, gender, work and address.

- Patients' past history.

- Clinico-radiological severity evaluated with WFNS (World Federation of Neurological Surgeons) and FISHER scores.

- Treatment: It is a protocol which is used in our Neurology Unit in case of SAH with the reason why surgical intervention was not realized.

- Patients' evolution during hospitalization: appearance of neurovascular complications and in-hospital mortality.

Those data were treated with $\mathrm{R}$ software. 
Study was done based on the confidentiality of information obtained from patients' records.

\section{Results}

During this period, we had 52 cases out of 2,656 hospitalized patients at $1.95 \% .37$ patients $(71.16 \%)$ were females with a sex ratio of $0: 40$. The mean age was between 44 and 48 years old with an extreme of 21 and 77 years old. Housewives $(n=14)$ were the most affected with $26 \%$ of cases. In $51.92 \%(n=27)$ of cases, our patients lived in urban areas, and had a past history of high blood pressure in $76.92 \%$ of cases. All patients had sudden headache with or without effort (Table I). In $51.92 \%$ of cases, aneurysm was in the anterior communicating artery. However, lack of financial means constitutes the main reason for the absence of surgical intervention $(71.15 \%)$. With the medical management,19.23\% (10) of patients had vasospasm and $40 \%$ did not have complications (Table I). Half of our patients had clinical severity more than WFNS grade III (Figure 1) and Fisher more than 3 in 39\% of cases (Figure 2).

During hospitalization, we had 7 deaths (13\%). With those who survived, $73 \%$ were valid and autonomous with modified ranking scale at 0 and $26 \%$. This also is with functional impairment with ranking scale at 4.

Table I. Characteristics of population study

\begin{tabular}{lll}
\hline Characteristics & $\begin{array}{l}\text { Total } \\
\text { number (n) }\end{array}$ & \% \\
\hline Age range & 24 & 13.47 \\
{$[20-50]$} & 28 & 30.77 \\
{$[50-80]$} & & \\
Profession & 11 & 21.15 \\
Office worker & 07 & 13.46 \\
Workers & 09 & 17.30 \\
Farmer & 14 & 26.92 \\
Housewives & 04 & 7.70 \\
Students & 07 & 13.46 \\
Unemployed & & \\
Residency & 27 & 51.92 \\
Urban & 18 & 34.61 \\
Suburban & 07 & 13.46 \\
Rural & & \\
Past history & 40 & 76.92 \\
High blood pressure & 04 & 7.70 \\
Oral contraception & 02 & 3.84 \\
Cranial trauma & 10 & 1.92 \\
Tobacco & 06 & 11.53 \\
Alcohol & 08 & 15.38 \\
None & & \\
Clinical manifestations & 39 & 75 \\
Sudden headache at rest & 13 & 25 \\
Sudden headache with effort & 48 & 92 \\
Repetitive throwing vomit & 32 & 61 \\
Photophonophobia & & \\
& &
\end{tabular}


Painful cervical stiffness

$\begin{array}{ll}45 & 86 \\ 25 & 48 \\ 10 & 19\end{array}$

Trouble of consciousness

86 19

Anterior communicating artery

Posterior communicating artery

Internal carotid

04

7.70

Middle cerebral artery

$04 \quad 7.70$

Basilar artery

02

3.84

Multiple

9.61

No surgical intervention because :

9.61

Patient or family refused

Lack of financial means

05

71.15

Insufficient interest (benefit/risk)

19.23

\section{Complications}

Vasospasm

Re-bleeding

Acute hydrocephalus

Trouble of consciousness

Seizures

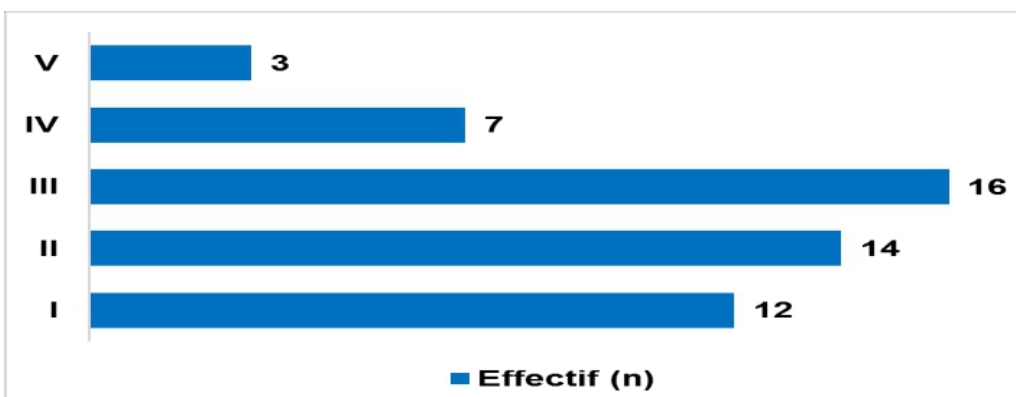

Figure 1. Severity classification of SAH according to the World Federation of Neurosurgeons (WFNS) scale

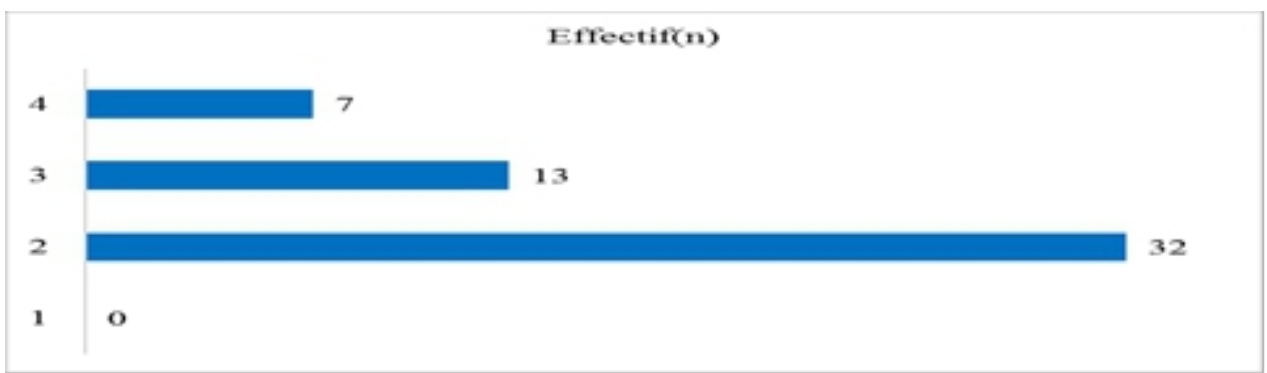

Figure 2. Repartition of patients according to radiological severity with the SAH scanography scale of Fisher

\section{Discussion}

In the beginning, we wanted to evaluate the performance of care and treatment applied in our country about aneurysmal SAH. Nevertheless, only 
12 patients had complete records (A surgical exclusion of an aneurysm with medical treatment). As a result, we adjusted our method and described the profile of patients who had SAH after disruption of aneurysm. This also includes those who do not undergo a surgical intervention and we described their evolution during hospitalization.

It is a monocentric study done in a reference center in Madagascar, but we cannot generalize the results.

About the population study, the access to surgical treatment of SAH is low in Madagascar with only $17 \%$ of patients $(n=12)$. It can be explained by the expensiveness of the treatment cost in regard to patients' financial means, and/or the place of recruitment which is a medical neurology unit.

We had 52 patients (1.96\%) admitted at our Neurology Unit. In Europe and North America, the global incidence reaches 9/1 and 100,000 habitants per year (Seiller et al., 2002). The difference is linked with care access and diagnosis means in those countries.

Our study found a ratio of 0:4 with 71\% females. Losseret et al found this same female predominance at $60 \%$. Thus, it can be explained by the role of hormonal factors. In fact, oestrogen acts in the vessel wall in preventing the collagen and elastin loss. A level 3 study proved that non menopause women had a lower risk of SAH instead of menopause ones with a risk relative of 0.24 and a confidence interval between 0.09-0.69 (Swiss SOS study group, 2015; Losseret et al., 2007).

Our population age was from 21-77 years old with a mean of 48 years old. In Morocco, Fedoul et al reported a pic estimated between 30-60 years old. In Switzerland, the mean age of patients with aneurysm SAH was about 54 years old. It can be explained by the precocious formation of aneurysm, the existence of its growth factor, and its rupture factor in young people such as alcoholism, tobacco, high blood pressure etc. (Swiss SOS study group, 2015; Fedoul et al., 2010).

As for profession, housewives represented $26 \%$ of patients. This, however, is because of the predominance of housewives in Malagasy people and those with SAH, especially women.

More than half of the patients live in urban areas (51.92\%). Hence, this reflects the repartition of the population because of the rural exodus in our country. In addition, the recruitment of our study was located in urban areas, in relation to the proximity of the patients' residence.

High blood pressure was reported in $76.92 \%$ of our patients. It is one of the risk factor clearly identified with smoking and alcohol. Contraception such as estroprogestative is one of the most discussed risk factor. Cocaine can be causal and seen as an aggravating factor also. Thus, it exists as a familial predisposition for SAH (Fedoul et al., 2010). 
Chronic high blood pressure can give micro-aneurysm of Charcot and Bouchard. Experimentally, it needs a minimal pressure at $155 \mathrm{mmHg}$ to start to develop an aneurysm. Brain vessel wall and the aneurysm are submitted by a pressure depending on systolic blood pressure. That is the way through which the rupture of an aneurysm is more frequent when blood pressure increases (Trehel, 2012; Michael et al., 2017).

Smoking also is a risk factor of SAH. It concerns active or weaning tabagic patients with respective relative risks at 61 and 27 compared to nonsmoking people. The mechanism why smoking increases the risk of SAH is not totally understood. However, it is plurifactorial (favors atherosclerosis in carotid artery, entails hemodynamic stress in Willis arterial circle...) if there is a preliminary intra-cranial aneurysm. Smoking increases the risk of rupture by increasing the pressure on aneurysm wall (sympathetic activation induced by nicotine, increasing of hematocrit rate and plasmatic fibrinogen) and in increasing local inflammation (Trehel, 2012; Michael et al., 2017; Gijn et al., 2007).

Chronic alcoholic intoxication constitutes a factor of SHA in case of high consummation (more than $150 \mathrm{~g}$ per week) with a relative risk of 2.1 , without significant difference with the two gender. The mechanism why alcohol increases the risk of SHA is not clearly demonstrated, but alcohol entails hemodynamic modification which can spoil vessel wall (Michael et al., 2017; Gijn et al., 2007).

All our patients had thunderclap headaches at rest or during daily efforts. It appears minutes after hemorrhage. Sometimes, headaches take place in many minutes and even many hours, but with extreme intensity. Headaches are due to inflammation and irritation of meninges by hemolyzed blood in subarachnoid spaces and infiltration of immunity cells, activation of immune system and pro-inflammatory cytokines. Headaches stay persistent with or without complications of SAH and are more intense if there are one or more complications (Curey-Levêque et al., 2014; Dufour et al., 2005).

Other symptoms can be associated such as, vomiting (70\%), seizures $(68 \%)$, and trouble of consciousness. Meningeal syndrome is an important sign but it appears many hours after $\mathrm{SAH}$, and it is not present at the time of admission (Curey-Levêque et al., 2014; Dufour et al., 2005).

In $50 \%$ of patients, WFNS scale was evaluated more. Many clinical scales that could evaluate the gravity of patients' state after SAH were described. The three most used scales are Hunt and Hess, World Federation of Neurological Surgeons (WFNS) scale, and Glasgow Coma Scale (GCS). The most reproducible element of appreciation which is more related to prognosis is GCS. It is included in WFNS scale, which has made certain authors to recommend only this scale (Seiller et al., 2002; Dufour et al., 2005; Hantson, 2012). 
Radiological aspects are a spontaneous hyper density in subarachnoid space on Braun CT scan without injection. The finding of aneurysm was done using Brain Angioscanner in our study, because arteriography and angioMRI are not available in our country. Due to the non-availability of the brain arteriography and angioMRI, Brain CT scan without injection remains the first exam for diagnosing SAH. Consequently, realized 12 hours after bleeding, its sensitivity reaches 98 to $100 \%$. This value decreases at $93 \%$ if done after 24 hours, and till 57 to $85 \%$ after the sixth day. Brain CT scan is also used in quantifying the $\mathrm{SAH}$, and then to establish a gradation by Fisher, which is a radiologic gravity scale that is predictive of vasospasm (Perdrix, 2011; Gauvrit et al., 2009).

FLAIR (Fluid Attenuated Inversion Recovery) sequence of MRI can detect SAH with a sensitivity of $100 \%$ in the first 2 weeks after bleeding, represented by hypersignal in subarachnoid spaces (Gauvrit et al., 2009).

Lumbar puncture can be done if imagery is negative. It presents typically a pink CSF with high concentration in red cells constant in all sample tubes, and also xanthochromy (Gijn et al., 2001).

Brain arteriography is the examination of reference to determine $\mathrm{SAH}$ etiology. This exam helps in determining the precise localization and the form to measure the size of aneurysm dome, as well as provide a view of the aneurysm neck. The research for other aneurysms is systematically done. A three dimensions reconstruction is frequently associated before therapeutic decision, allowing a precise analysis of the aneurysm neck, dome, and the aneurysm adjacent vascular structures (Gauvrit et al., 2009; Gijn et al., 2001).

$51.92 \%$ aneurysm was localized in the anterior communicating artery in our study. It can be explained by the high turbulence in the bifurcation of the anterior circulation. Its location is in the anterior communicating artery in 30 of the cases and can be multiples in 20\%-30\% of cases (HAS, 2009).

We hospitalized patients who did not undergo surgery of their aneurysm during 21 days in our emergency unit. It is, however, the protocol of our unit to prevent complications which can be present during this period. It also allows us to watch over them and carry out immediate ripostes. Management insists on a strict bed rest during 21 days to prevent re-bleeding, without moral or physic effort.

Surgical intervention was not done in our patients because of financial problems in $71.15 \%$; the absence of surgical indication after discussion about the benefit/risk rapport in 19,23\%; and patients with/or their family refusal of surgery and demand just a medical treatment in $9.61 \%$.

According to literature, a part of general measure, particularly strict bed rest, continued monitoring and frequent neurologic medical supervision by the Glagow scale, systematic IV analgesic treatment and adapted by EVA 
scale, and the prevention of stress ulcerous by OMEPRAZOLE. The anticomitial treatment by CLONAZEPAM IV is done per day if there are seizures.

Aneurysm exclusion should be done by surgical or endovascular means within 24 to 72 hours after admission. This exclusion should be complete and definitive and controlled by angiographic means (digitalized angiography or angioscanner) after the procedure (Alejandro et al., 2012; Aisiku et al., 2014; Edlow et al., 2012).

Furthermore, those differences of management are due to the difference of socio-economic and demographic data of our country compared to developed countries.

In absence of surgical treatment of the intracranial aneurysm, $19.23 \%$ of patients had vasospasm, $11.53 \%$ bled again, $15.38 \%$ had seizures with other complications such as acute hydrocephalus, status epilepticus and trouble of consciousness, and $40.38 \%$ of patients had no complication.

- Arterial vasospasm can be met in $25 \%$ cases and occurs on the 5th and the 12th day. Arteries which are in the hemorrhagic region will present sustained vasoconstriction leading eventually to cerebral infraction in those vascular territories (Leblanc et al., 2013; Berré et al., 2005).

- Re-bleeding is frequent and worse than the initial bleeding with aneurysm. Its frequency decreases with the time compared to initial bleeding at $4 \%$ the first day, 20-30\% during the first month (with a pic of frequency of the 7 th and the 14th day), and 50\% during the first six months. In addition, beyond the sixth month, re-bleeding risk is at 3\% per year. Mortality linked to re-bleeding is high (about $10 \%$ ) (Proust et al., 2005).

- Acute hydrocephalus is linked especially with intra-ventricular hemorrhage, which entails an obstruction of Sylvius aqueduct and an intracranial hypertension (with tri-ventricular dilatation on brain CT scan), which needs an emergency external ventricular derivation (Berré et al., 2005; Proust et al., 2005).

During those 21 days of hospitalization, we deplored 7 deaths (13\%) and 45 survivors $(87 \%)$. Mortality rate stays high in hospital areas, reaching $50 \%$ of patients on an average. Other authors reported a mortality rate around 20-35\% of hospitalized patients.

This discordance, on one hand, can be explained by the care protocol of our unit. Therefore, this is done based on complication prevention in case of the impossibility of surgical treatment.

On another hand, surgical treatments are not free of complication. As a result, it increases the mortality rate as described below:

- Disruption of aneurysmal bag while aneurysm is not yet accessible may be responsible for $\mathrm{SAH}$ recurrence which can be accompanied by high morbi-mortality rate; 
- Ischemic complication by occlusion of the artery which carries the aneurysm or one of its branches when neurosurgical clip is put;

- Infectious risks (post-surgical meningitis, abscess);

- Neuro-physiologic complications notably during the dissection of perforating artery of frontal lobe during anterior communicating artery aneurysm surgery (Grasso et al., 2017; Leblanc et al., 2000; Steiner et al., 2013).

\section{Conclusion}

In our study, in majority of cases, patients who had SAH are young females and housewives living in urban areas. This was accompanied by anterior communicating artery aneurysm and a sudden headache. In Madagascar, access to surgical treatment is limited for patients with SAH. Our study shows this technical failure based on the diagnosis and treatment of SAH. Even the data of our study stays limited. However, the efficiency of our unit treatment protocol was proven. We had better issues in terms of complications of in-hospital death and dependence at discharge. To sum it up, an evaluative study is needed to establish the real efficiency of this management, and treatment abstention should also be put into consideration.

\section{References:}

1. Aisiku, I., Edlow, JA., Goldstein, J., \& Thomas, LE. (2014). An Evidence-Based Approach To Diagnosis And Management Of Subarachnoid Hemorrhage In The Emergency Department. EB Medicine ; 16 (10) : 1 - 32.

2. Alejandro, SCE., Rabinstein, A., Carhuapoma J., Colin, R., Derdeyn, P., \& Dion, J., et al. (2012). Guidelines for the Management of Aneurysmal Subarachnoid Hemorrhage ; Stroke ; 43 : 1- 27.

3. Berré, J., Gabrillargues, J., Audibert, G., Hans, P., Bonafé, A., Boulard, G., et al. (2005). Hémorragies méningées graves : prévention, diagnostic et traitement $\mathrm{du}$ vasospasme. Annales Françaises d'Anesthésie et de Réanimation ; 24 : 761-774.

4. Curey-Levêque, S., Gilles Baray, M., Ozkul-Wermester, O., Fréger, P., Proust, F. (2014). Hémorragies sous - arachnoïdiennes non traumatiques. EMC Neurologie ; $17-152-\mathrm{A}-10$.

5. Dufour, H., Bonafé, A., Bruder, N., Boulard, G., Ravussin, P., Lejeune, JP., et al. (2005). Diagnosis in general hospital and immediate care of patients suffering from severe subarachnoid haemorrhage. Annales Françaises d'Anesthésie et de Réanimation ; 24 : 715-720.

6. Edlow, JA. \& Fisher, J. (2012). Diagnosis of Subarachnoid Hemorrhage Time to Change the Guidelines? Stroke ; 43 ; 2031-2032. 
7. Fedoul, B., Chakour, K., \& Chaoui, ME. (2010). L'hémorragie méningée par rupture anévrysmale. Annales de Médecine et de Thérapeutique ; 2 (1) : $66-73$.

8. Gauvrit, JY., Leclerc, X., Ferré, JC., Taschner, CA., Carsin-Nicol, B., Aufray-Calvier, E., Morandi, X., \& Carsin, M. (2009). Imagérie de l'hémorragie sous arachnoidienne. Journal of neuroradiology ; $36: 65$ -73 .

9. Gijn, JV., Kerr RS., \& Rinkel, GJE. (2007). Subarachnoid haemorrhage. Lancet ; $369: 306-318$.

10. Gijn, JV. \& Rinkel, GJE. (2001). Subarachnoid hemorrhage : diagnosis, causes and management. Brain; $124: 249-276$.

11. Grasso, G., Alafaci, C., \& Macdonald, RL. (2017). Management of aneurysmal subarachnoid hemorrhage : State of the art and future perspectives. Surg Neurol Int ; $8: 11$.

12. Hantson, P. (2012). Physiopathologie des lésions cérébrales précoces et retardées dans l'hémorragie sous-arachnoïdienne : avancées récentes. Réanimation ; 21 : S475-S481.

13. Haute Autorité de Santé (HAS). (2009). Actualisation du rapport d'évaluation sur les anévrismes sacculaires intracrâniens rompus : occlusion par voie endovasculaire versus exclusion par microchirurgie $; 1-85$.

14. Leblanc, PE. \& Engrand, N. (2000). Hemorragie meningée postanevrysmale : role de l'anesthesiste-reanimateur. MAPAR ; 478 - 488.

15. Leblanc, PE., Le Maho, AL., \& Vigué, B. (2013). Hémorragies méningées en 2013 : aller au-delà du vasospasme. MAPAR ; 438 446.

16. Losser, MR. \& Payen, D. (2007). Management of subarachnoid hemorrhage. Réanimation ; $16: 463-471$.

17. Michael, T., Lawton, G., \& Vates, E. (2017). Subarachnoid Hemorrhage. N Engl J Med ; 377 : 257 - 266.

18. Perdrix, J. (2011). Hémorragie sous-arachnoïdienne : sensibilité du scanner cérébral. Revue Médicale Suisse ; 1724 - 1725.

19. Proust, F., Bonafé, A., Lejeune, JP., De Kersaint-Gilly, A., Gabrillargues, J., Dufour, H., et al. (2005). L'anévrisme : occlure le sac pour prévenir le resaignement. Annales Françaises d'Anesthésie et de Réanimation ; 24 : 746-755.

20. Seiller, N., Apostu, VI., Pavy-Le-Traon, A., Arrue, P., Lagarrigue, J., \& Geraud, G. (2002). Hémorragies méningées. Encycl Med Chir Neurologie ; 17-152-A-10.

21. Steiner, T., Juvela, S., Unterberg, A., Jung, C., Forsting, M., \& Rinkel, G. (2013). Guidelines for the Management of Intracranial Aneurysms and Subarachnoid Haemorrhage. Cerebrovasc Dis ; $35: 93-11$. 
22. Swiss SOS study group. (2015). Evaluation neuropsychologique dans l'hémorragie sous-arachnoïdienne anévrismale. Forum Médical Suisse ; 15(48) :1122-1127.

23. Trehel, V. (2012). Hémorragie sous arachnoïdienne du sujet âgé : L'hydrocéphalie à l'admission comme marqueur de mauvais pronostic. Thèse de doctorat en Anesthésie et Réanimation. Nº617751, Faculté de médecine de Créteil ; 5 - 60 . 